\title{
A Double-Blind, Randomized Pilot Trial of Chromium Picolinate for Overweight Individuals with Binge-Eating Disorder: Effects on Glucose Regulation
}

\author{
Margarita Sala, BS ${ }^{1,2}$, Lauren Breithaupt, $\mathrm{MA}^{1,3}$, Cynthia M. Bulik, $\mathrm{PhD}^{1,4,5}$, \\ Robert M. Hamer, $\mathrm{PhD}^{1}$, Maria C. La Via, $\mathrm{MD}^{1}$, \& Kimberly A. Brownley, $\mathrm{PhD}^{1}$ \\ ${ }^{1}$ Department of Psychiatry, University of North Carolina at Chapel Hill, Chapel Hill, \\ North Carolina, USA, ${ }^{2}$ Department of Psychology, Southern Methodist University, \\ Dallas, Texas, USA, ${ }^{3}$ Department of Psychology, George Mason University, Fairfax, \\ Virginia, USA, ${ }^{4}$ Department of Nutrition, University of North Carolina at Chapel Hill, \\ Chapel Hill, North Carolina, USA, ${ }^{5}$ Department of Medical Epidemiology and \\ Biostatistics, Karolinska Institutet, Stockholm, Sweden
}

\begin{abstract}
Purpose: Chromium treatment has been shown to improve glucose regulation in some populations. The purpose of this study was to evaluate whether chromium picolinate (CrPic) supplementation improves glucose regulation in overweight individuals with binge-eating disorder (BED). Methods: In this double-blinded randomized pilot trial, participants $(N=24)$ were randomized to high $(\mathrm{HIGH}, 1000 \mathrm{mcg} / \mathrm{day}, n=8)$ or moderate (MOD, $600 \mathrm{mcg} / \mathrm{day}, n=9$ ) dose of CrPic or placebo (PL, $n=7)$ for 6 months. Participants completed an oral glucose tolerance test (OGTT) at baseline, 3 months, and 6 months. Fixed effects models were used to estimate mean change in glucose area under the curve (AUC), insulin ${ }_{\mathrm{AUC}}$, and insulin sensitivity index (ISI). Results: Results revealed a significant group and time interaction $(p<0.04)$ for glucose AUC $_{\text {, }}$, with glucose $_{\text {AUC }}$ increasing significantly in the PL group $(p<0.02)$ but decreasing significantly in the MOD group $(p<0.03)$ at 6 months. Insulin AUC $_{\text {increased significantly }}$ over time (main effect, $p<0.02$ ), whereas ISI decreased significantly over time (main effect, $p<0.03$ ). Conclusion: As anticipated, a moderate dose of $\mathrm{CrPic}$ was associated with improved glycemic control, whereas PL was associated with decreased glycemic control. It was unexpected that the improved glycemic control seen in the MOD dose group was not seen in the HIGH dose group. However, although participants randomized to the HIGH dose group did not have improved glycemic control, they had better glycemic control than participants randomized to the PL group. These findings support the need for larger trials.
\end{abstract}

KEYWORDS. alternative medicine, binge-eating disorder, chromium, glucose 
Binge-eating disorder (BED) is a psychiatric disorder that has recently been included in the Diagnostic and Statistical Manual of Mental Disorders, Fifth Edition (DSM-5) as an independent eating disorder diagnosis for the first time (American Psychiatric Association 2013). BED is characterized by recurrent episodes of binge eating, defined as the consumption of an unusually large amount of food in a short period of time accompanied with a feeling of loss of control over eating, in the absence of inappropriate compensatory behaviors. BED is the most prevalent eating disorder, affecting $3.5 \%$ of adult women and $2 \%$ of adult men in the U.S. (Hudson et al., 2007). BED is highly comorbid with several psychiatric and medical conditions, including depression, metabolic syndrome (i.e., meeting at least three risk factors for arthosclerotic cardiovascular disease), and diabetes (Reichborn-Kjennerud et al., 2004, Yanovski et al., 1993, Bulik, Sullivan, \& Kendler, 2002, Grilo, White, \& Masheb, 2009). Notably, individuals with BED have significantly greater risk of developing components of the metabolic syndrome (e.g., abdominal obesity, dyslipidemia, hypertension, and abnormal glucose metabolism) independent of the risk of obesity alone and type 2 diabetes (T2DM) than individuals without BED (Hudson et al., 2010). Individuals with T2DM who also struggle with binge eating and/or depression have worse diabetes-related complications and poorer diabetes outcomes (Rotella et al., 2012, de Groot et al., 2001, Katon et al., 2009, Lin et al., 2010). For example, in one study of individuals with T2DM, eating pathology was associated with unsatisfactory glycemic control (Rotella et al., 2012).

Currently available options for BED treatment include several psychological and pharmacological interventions (Berkman et al., 2015; Brownley et al., 2016). Although some of these interventions are effective in helping patients reduce binge eating and its related psychological distress and physical consequences, not all patients respond adequately in terms of achieving binge abstinence and mood and metabolic stabilization. BED has historically been treated with medications such as citalopram, fluoxetine, fluovoxamine, imipramine, orlistat, sertraline, sibutramine, and more recently lixdexamfetamine (McElroy et al. 2015; McElroy et al., 2016). However, many patients drop out of pharmacological BED treatment due to medication side effects. Side effects associated with these medications in the treatment of BED include blurred vision, constipation, decreased libido, depression, dizziness, dry mouth, fatigue, headache, increased urinary frequency, insomnia, nausea, nervousness, sexual dysfunction, sedation, and somnolence (Brownley et al., 2007). Of concern, little information exists regarding the treatment and prevention of T2DM in individuals with BED. Although lifestyle interventions are effective, they are difficult to sustain (Korkiakangas, Alahuhta, \& Laitinen, 2009). Drug treatments for T2DM have numerous side effects and limited durability. Addressing these unmet treatment needs in BED requires further investigation of novel interventions that ultimately help patients achieve binge eating abstinence and healthy weight and metabolic stabilization.

Chromium is an essential mineral that affects insulin and serotonin functioning and has the potential to also influence dopamine functioning (Davis \& Vincent, 1997, McCarty, 1994), making it a plausible candidate for treatment of the complex patient with comorbid BED, depression, and T2DM. Prior studies have shown that chromium supplementation attenuates weight gain and visceral fat ac- 
cumulation in patients with T2DM and improves insulin sensitivity and glycemic control in patients with T2DM, metabolic disorder, and polycystic ovary syndrome (Martin et al., 2006, Albarracin et al., 2008). In addition, chromium supplementation reduced carbohydrate cravings and appetite in patients with atypical depression (Docherty et al., 2005). The dose of chromium studied affects treatment efficacy, although the optimal dosage level is unknown (Broadhurst \& Domenico, 2006). Building on these findings and our understanding of the physiological and psychological components of BED, the purpose of the present study was to evaluate whether a high or moderate dose of chromium supplementation improves glucose regulation in overweight individuals with BED. We hypothesized that high and moderate doses of chromium supplementation would be associated with improved glycemic control, as measured by their response to an oral glucose tolerance test (OGTT).

\section{METHODS}

\section{Participants}

A total of 220 potential participants self-referred to the study, of whom 43 passed screening and 41 met initial eligibility criteria (i.e., age between 18 and 60, DSMIV criteria for BED, not pregnant or lactating, not currently engaged in other BED treatments or using chromium). Out of the 41 patients assessed, three declined to participate, and 10 met exclusion criteria. The remaining 28 eligible participants met DSM-IV criteria for BED and all other inclusion criteria. Inclusion and exclusion criteria are outlined in Table 1 . This study was approved by the Institutional Review Board at the University of North Carolina at Chapel Hill.

\section{Procedure}

The 28 eligible participants underwent a 1-month placebo run-in, after which four participants were excluded due to placebo response. The remaining participants

\section{TABLE 1. Inclusion and exclusion criteria}

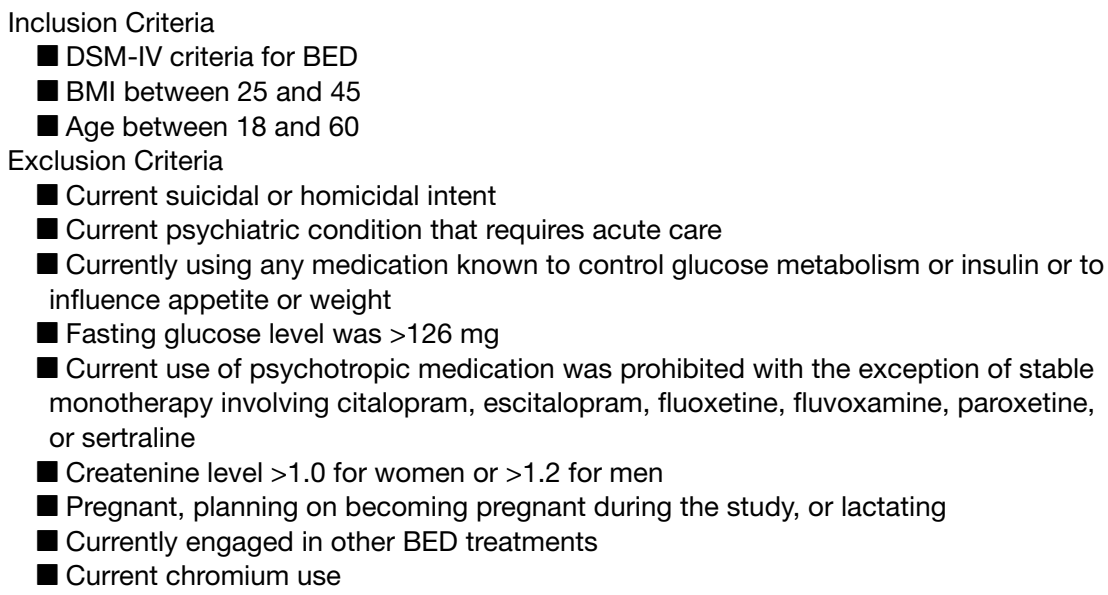

Notes: DSM-IV = Diagnostic and Statistical Manual of Mental Disorders, Fourth Edition; BED = Binge-Eating Disorder. 
were randomized to HIGH (1000 mcg/day, $\mathrm{n}=8)$ or MOD (600 mcg/day, $\mathrm{n}=9$ ) dose of CrPic or PL $(n=7)$ for 6 months. The CrPic doses were chosen in light of previous studies with non-BED populations. Specifically, a moderate dose of 600 $\mathrm{mcg}$ /day was chosen, as literature suggests that this level of dosage would be sufficient for improving fasting glucose, insulin binding, and reducing depressive symptoms and cravings (Anderson et al., 1987, Davidson et al., 2003, Docherty et al., 2005). A high dose of $1,000 \mathrm{mcg} / \mathrm{day}$ was chosen, as the literature suggests that this level of dosage is needed to prevent weight gain in subjects with T2DM (Martin et al., 2006). Adherence was assessed by asking participants to return all unused pills at each study visit.

Participants who were randomized into the study completed an OGTT at baseline, 3 months, and 6 months. Prior to each OGTT, participants fasted overnight. For the OGTT, a glucose solution standardized for body weight at $1.75 \mathrm{~g} / \mathrm{kg}$ was consumed. Blood samples were obtained via an intravenous catheter at minute 0 (fasted) and then minutes 30, 60, 90, and 120 after ingestion of the glucose solution. Nineteen participants completed all study visits, with four participants withdrawing from the study due to personal reasons and one due to pregnancy.

Glucose was assayed with an Ortho Clinical Diagnostics Vitros 950 analyzer (UNC Hospitals). Insulin was measured using a competitive radioimmunoassay (Diagnostic Systems Labs, Webster, TX) at the UNC Endocrine Lab. Assay sensitivity was $1.3 \mu \mathrm{IU} / \mathrm{mL}$ with a standard range of 5-300 $\mu \mathrm{U} / \mathrm{mL}$. Glucose and insulin area under the curve (AUC) were calculated using the trapezoidal rule. ISI was evaluated by the formula: $10,000 / \sqrt{ }$ (fasting glucose $\times$ fasting insulin $\times$ mean glucose $\times$ mean insulin) (Matsuda \& DeFronzo, 1999). Fixed effect models with a random intercept were used to estimate the mean change in glucose AUC $_{\text {, }}$, insulin ${ }_{\mathrm{AUC}}$, and ISI over the 3-month and 6-month active treatment period.

\section{RESULTS}

\section{Characteristics of the Sample}

Our sample included 24 randomized participants. The mean age of the sample was 36.6 (SD 10.6) years and their mean BMI was $34.2 \mathrm{~kg} / \mathrm{m}^{2}$ (SD 5.4). Most of the sample was white $(87.5 \%)$ and female $(83.8 \%)$. Participants exhibited a mild level of depression, as measured by the Quick Inventory of Depressive Symptomatology Self-Report [mean (SD) = 6.8 (3.8)] and were nondiabetic [mean (SD) fasting plasma glucose $=90.3(9.4) \mathrm{mg} / \mathrm{dL}]$. The mean HbA1c of the sample was 5.5. There were no statistically significant differences between the HIGH, MOD, and PL groups on any of these measures.

\section{Outcomes}

Figures 1-3 depict the results of our study. The analyses revealed a statistically significant group and time interaction $(p<0.04)$ for glucose AUC $_{\text {, with a statically }}$ significant increase in the PL group at 6 months $(p<0.02)$, but a statistically significant decrease at 6 months in the MOD group $(p<0.03)$. From baseline to 6 months, glucose $\mathrm{AUC}_{\mathrm{AU}}$ increased $12.6 \%$ in the PL group, decreased $10.1 \%$ in the MOD dose group, and increased $6.5 \%$ in the $\mathrm{HIGH}$ dose group. Insulin AUC $_{\text {increased }}$ 


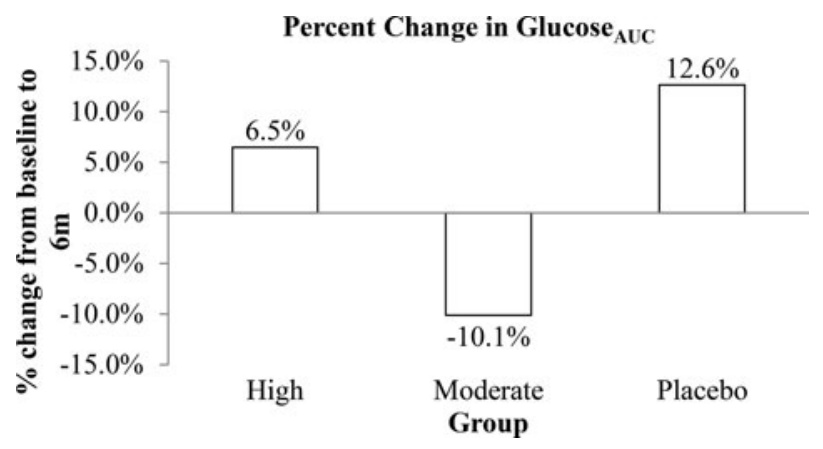

FIGURE 1. Percent change in glucose $\mathrm{AUC}_{\mathrm{AUC}}$ from baseline to 6 months. $\mathrm{AUC}=$ area under the curve.

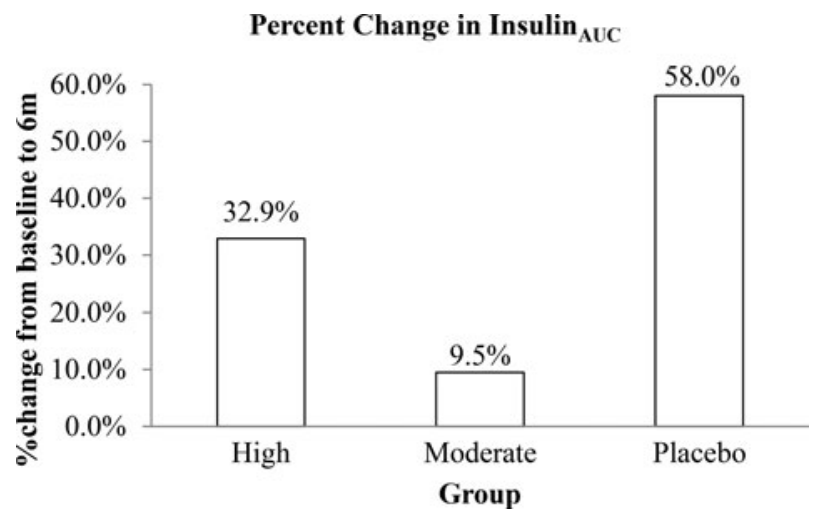

FIGURE 2. Percent change in insulin ${ }_{\mathrm{AUC}}$ from baseline to 6 months. AUC $=$ area under the curve.

significantly over time (main effect, $p<0.02$ ). From baseline to 6 months, insulin $_{\mathrm{AUC}}$ increased $58.0 \%$ in the PL group, increased $9.5 \%$ in the MOD dose group, and increased $32.9 \%$ in the HIGH dose group. ISI decreased significantly over time (main effect, $p<0.03$ ). From baseline to 6 months, ISI decreased $42.6 \%$ in the PL group, increased $13.6 \%$ in the MOD dose group, and decreased $24.1 \%$ in the HIGH dose group.

\section{DISCUSSION}

The purpose of this study was to evaluate the effect of CrPic supplementation on glucose regulation in patients with BED. In this small sample, a moderate dose of CrPic supplementation was associated with improved glycemic control, whereas a PL dose was associated with decreased glycemic control. Specifically, glucose $\mathrm{AUC}$ decreased in the moderate dose group but increased in the placebo group. All participants and particularly those in the placebo group experienced an increase in insulin $_{\mathrm{AUC}}$. ISI decreased significantly over time, with the largest magnitude decrease occurring in the PL group. 


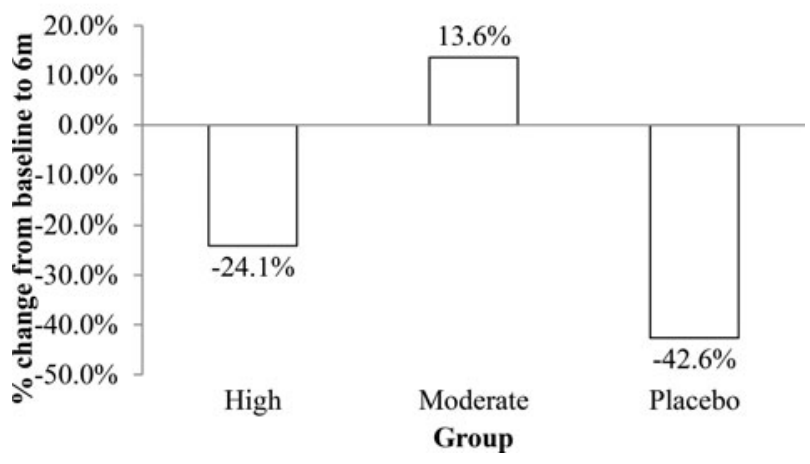

FIGURE 3. Percent change in ISI from baseline to six months. ISI = insulin sensitivity index.

Glucose $_{\text {AUC }}$ increased in the PL group but decreased in the MOD group over time. The significant decrease in the MOD group is consistent with other chromium supplementation studies (Martin et al., 2006). However, it was unexpected that the improved glycemic control seen in the MOD dose group was not seen in the HIGH dose group. One explanation for this is that the individuals in the moderate group could have experienced optimal improvement in glycemic control because they had the highest Hba1c levels to begin with, as previous research has shown that chromium provides the greatest benefits to individuals with higher Hba1c levels (Albarracin et al., 2008). Alternatively, the results could have occurred due to differences in binge frequency. The HIGH group had the highest number of binge episodes at baseline. Although the HIGH group experienced a reduction in binge frequency over the course of the trial, their number of binge episodes at the end of treatment were still not as low as that of the MOD group. Finally, it is possible that this unexpected finding occurred due to the limited statistical power of this study. Additional adequately powered studies are warranted to evaluate chromium supplementation for glycemic control in individuals with BED who are at increased risk of metabolic disease. However, it is important to note that the magnitude of increase was larger in the PL group than in the HIGH dose group.

Insulin $_{\mathrm{AUC}}$ increased in all groups, with the largest magnitude increase occurring in the placebo group. The overall increase in insulin ${ }_{\mathrm{AUC}}$ was also found in another study evaluating chromium supplementation in individuals with T2DM (Martin et al., 2006). The particularly high increase in insulin ${ }_{\mathrm{AUC}}$ in the placebo group is evidence for worsening metabolic disease states in untreated patients with BED, which is consistent with a longitudinal study on patients with BED conducted by Hudson and collaborators (2010).

ISI decreased significantly over time, with the largest magnitude decrease occurring it the placebo group. These results differ from the results of another study, which found that CrPic supplementation has no effect on insulin sensitivity in obese nondiabetic adults (Iqbal et al., 2009). It is possible that CrPic supplementation has an effect on insulin sensitivity in some populations but not others.

Limitations of this study include its small and homogeneous self-referred sample, the lack of an indicator of compliance (e.g., urinary chromium levels), and the fact 
that the entire study was conducted in a single center. Despite limitations, this was the first trial to evaluate different doses of $\mathrm{CrPic}$ on the improvement of glucose regulation in individuals with BED.

In summary, this double-blind placebo-controlled six month pilot trial evaluated whether CrPic supplementation improves glucose regulation in individuals with BED. A $600 \mathrm{mcg} /$ day dose of CrPic was correlated with improved glycemic control. On average, participants experienced an increase in insulin AUC $_{\text {and a decrease }}$ in ISI. The largest insulin ${ }_{\mathrm{AUC}}$ increase and the largest ISI decrease occurred the PL group. However, our sample was small and future adequately powered studies are warranted.

Declaration of Interest: Dr. Bulik is a grant recipient from and a consultant for Shire and has consulted for Ironshore.

\section{FUNDING}

This study was supported by a grant from the Brain \& Behavior Foundation (formerly NARSAD) to Dr. Kimberly Brownley. Lauren Breithaupt and Margarita Sala are supported by the National Science Foundation Graduate Research Fellowship under Grant No. DGE-1356109. Any opinion, findings, and conclusions or recommendations expressed in this material are those of the authors(s) and do not necessarily reflect the views of the National Science Foundation. Dr. Cynthia Bulik acknowledges funding from the Swedish Research Council (VR Dnr: 5382013-8864).

\section{ABOUT THE AUTHORS}

Margarita Sala is a Clinical Psychology PhD student at Southern Methodist University in Dallas, Texas. Lauren Breithaupt is a Clinical Psychology PhD student at George Mason University in Fairfax, Virginia. Cynthia M. Bulik, PhD, is Distinguished Professor of Eating Disorders in the Department of Psychiatry and Professor of Nutrition at the University of North Carolina at Chapel Hill. She is also Professor of Medical Epidemiology and Biostatistics at Karolinska Institutet, Stockholm, Sweden. Robert M. Hamer, PhD, was Professor of Psychiatry and Biostatistics at the University of North Carolina at Chapel Hill at the time of his untimely death on December 28, 2015. Maria C. La Via, MD, is Associate Professor in the Department of Psychiatry at UNC Chapel Hill. Kimberly A. Brownley is Associate Professor of Psychiatry at the University of North Carolina at Chapel Hill, USA. Her research focuses on the psychobiology of appetite regulation including the role of appetite-regulating gut peptides in weight and mood dysregulation in psychiatric populations. She served as Principal Investigator on the present study and lead author on the parent article that was published in 2013 (Brownley et al., 2013). 


\section{REFERENCES}

Albarracin CA, Fuqua BC, Evans JL, Goldfine ID. Chromium picolinate and biotin combination improves glucose metabolism in treated, uncontrolled overweight to obese patients with type 2 diabetes. Diabetes Metab Res Rev. 2008;24(1):41-51. doi:10.1002/dmrr.755.

American Psychiatric Association. Diagnostic and statistical manual of mental disorders (5th ed.). Washington, DC, 2013.

Anderson RA, Polansky MM, Bryden NA, Bhathena SJ, Canary JJ. Effects of supplemental chromium on patients with symptoms of reactive hypoglycemia. Metabolism. 1987;36(4):351-355. doi:10.1016/0026-0495(87)90206-X.

Berkman ND, Brownley KA, Peat CM, Lohr KN, Cullen KE, Morgan LC, Bann CM, Wallace IF, Bulik CM. Management and Outcomes of Binge-Eating Disorder [Internet]. Rockville (MD): Agency for Healthcare Research and Quality (US); 2015 Dec. PMID:26764442.

Broadhurst CL, Domenico P. Clinical studies on chromium picolinate supplementation in diabetes mellitus-A review. Diabetes Technol Ther. 2006;8(6):677-687. doi:10.1089/dia. 2006.8.677.

Brownley KA, Berkman ND, Sedway JA, Lohr KN, Bulik CM. Binge eating disorder treatment: a systematic review of randomized controlled trials. Int J Eat Disorder. 2007;40(4):337-348. doi:10.1002/eat.20370.

Brownley KA, Berkman ND, Peat CM, Lohr KN, Cullen KE, Bann CM, Bulik CM. Binge-Eating Disorder in Adults: A Systematic Review and Meta-analysis. Ann Intern Med. 2016 Jun 28. doi: 10.7326/M15-2455. [Epub ahead of print] PMID:27367316.

Brownley KA, Von Holle A, Hamer RM, La Via M, \& Bulik CM. A double-blind, randomized pilot trial of chromium picolinate for binge eating disorder: results of the Binge Eating and Chromium (BEACh) study. Journal of Psychosomatic Research, 2013;75(1):36-42.

Bulik CM, Sullivan PF, Kendler KS. Medical and psychiatric morbidity in obese women with and without binge eating. Int J Eat Disorder. 2002;32(1):72-78. doi:10.1002/eat.10072.

Davidson JR, Abraham K, Connor KM, McLeod MN. Effectiveness of chromium in atypical depression: a placebo-controlled trial. Biol. Psychiatry. 2003;53(3):261-264. doi:10.1016/S00063223(02)01500-7.

Davis CM, Vincent JB. Chromium oligopeptide activates insulin receptor tyrosine kinase activity. Biochemistry. 1997;36(15):4382-4385. doi:10.1021/bi963154t.

de Groot M, Anderson R, Freedland KE, Clouse RE, Lustman PJ. Association of depression and diabetes complications: a meta-analysis. Psychosom Med. 2001;63(4):619-630. doi: 10.1097/00006842-200107000-00015.

Docherty JP, Sack DA, Roffman M, Finch M, Komorowski JRA. Double-blind, placebocontrolled, exploratory trial of chromium picolinate in atypical depression: effect on carbohydrate craving. J.Psychiatr Pract. 2005;11(5):302-314. doi: 10.1097/00131746-200509000-00004.

Grilo CM, White MA, Masheb RM. DSM-IV psychiatric disorder comorbidity and its correlates in binge eating disorder. Int J Eat Disorder. 2009;42(3):228-234. doi:10.1002/eat.20599.

Hudson JI, Hiripi E, Pope HG, Jr., Kessler RC. The prevalence and correlates of eating disorders in the National Comorbidity Survey Replication. Biol. Psychiatry 2007;61:(3):348-358. doi:10.1016/j.biopsych.2006.03.040.

Hudson JI, Lalonde JK, Coit CE, Tsuang MT, McElroy SL, Crow SJ, et al. Longitudinal study of the diagnosis of components of the metabolic syndrome in individuals with binge-eating disorder. Am J Clin Nutr. 2010;91(6):1568-1573. doi:10.3945/ajen.2010.29203.

Iqbal N, Cardillo S, Volger S, Bloedon LT, Anderson RA, Boston R, et al. Chromium picolinate does not improve key features of metabolic syndrome in obese nondiabetic adults. Metab Syndr Relat Disord. 2009;7(2):143-150. doi: 10.1089/met.2008.0048.

Katon W, RussoJ, Lin EH, Heckbert SR, Karter AJ, Williams LH, et al. Diabetes and poor disease control: is comorbid depression associated with poor medication adherence or lack of treatment intensification? Psychosom Med. 2009;71(9):965-972. doi:10.1097/PSY.0b013e3181bd8f55.

Korkiakangas EE, Alahuhta MA, Laitinen JH. Barriers to regular exercise among adults at high risk or diagnosed with type 2 diabetes: a systematic review. Health Promot Int. 2009;24(4):416-427. doi:10.1093/heapro/dap031. 
Lin EH, RutterCM, Katon W, Heckbert SR, Ciechanowski P, Oliver MM, et al. Depression and advanced complications of diabetes: a prospective cohort study. Diabetes Care. 2010;33(2):264-269. doi:10.2337/dc09-1068.

Martin J, Wang ZQ, Zhang XH, Wachtel D, Volaufova J, Matthews DE, et al. Chromium picolinate supplementation attenuates body weight gain and increases insulin sensitivity in subjects with type 2 diabetes. Diabetes Care. 2006;29(8):1826-1832. doi:10.2337/dc06-0254.

Matsuda M, DeFronzo RA. Insulin sensitivity indices obtained from oral glucose tolerance testing: comparison with the euglycemic insulin clamp. Diabetes Care. 1999;22(9):1462-1470. doi: 10.2337/diacare.22.9.1462.

McCarty MF. Enhancing central and peripheral insulin activity as a strategy for the treatment of endogenous depression-an adjuvant role for chromium picolinate? Med Hypotheses. 1994;43(4):247-252. http://dx.doi.org/10.1016/0306-9877(94)90075-2.

McElroy SL, Hudson J, Ferreira-Cornwell MC, Radewonuk J, Whitaker T, Gasior M. Lisdexamfetamine Dimesylate for Adults with Moderate to Severe Binge Eating Disorder: Results of Two Pivotal Phase 3 Randomized Controlled Trials. Neuropsychopharmacology. 2016 Apr;41(5):1251-60. doi: 10.1038/npp.2015.275. Epub 2015 Sep 9. PMID:26346638.

McElroy SL, Hudson JI, Mitchell JE, Wilfley D, Ferreira-Cornwell MC, Gao J, Wang J, Whitaker T, Jonas J, Gasior M. Efficacy and safety of lisdexamfetamine for treatment of adults with moderate to severe binge-eating disorder: a randomized clinical trial. JAMA Psychiatry. 2015 Mar;72(3):235-46. doi: 10.1001/jamapsychiatry.2014.2162. PMID:25587645.

Reichborn-Kjennerud T, Bulik CM, Sullivan PF, Tambs K, Harris JR. Psychiatric and medical symptoms in binge eating in the absence of compensatory behaviors. Obes Res. 2004;12(9):1445-1454. doi:10.1038/oby.2004.181.

Rotella F, Cresci B, Monami M, Aletti V, Andreoli V, Ambrosio ML, et al. Are psychopathological features relevant predictors of glucose control in patients with type 2 diabetes? A prospective study. Acta Diabetol. 2012;49(Suppl 1):S179-184. doi:10.1007/s00592-012-0403-4.

Yanovski SZ, Nelson JE, Dubbert BK, Spitzer RL. Association of binge eating disorder and psychiatric comorbidity in obese subjects. Am. J. Psych. 1993;150(10):1472-1479. 